\title{
Chaos synchronization delay in semiconductor lasers with
}

\section{optoelectronic feedback}

\author{
Atyaf Adnan, Kais Al-Naimee, Sudad S. Ahmed
}

Department of Physics, College of Science, University of Baghdad, Baghdad, Iraq

E-mail: maryam adnan212@yahoo.com

\begin{abstract}
In this work we reported the synchronization delay in semiconductor laser (SL) networks. The unidirectional configurations between successive oscillators and the correlation between them are achieved. The coupling strength is a control parameter so when we increase coupling strength the dynamic of the system has been change. In addition the time required to synchronize network components (delay of synchronization) has been studied as well. The synchronization delay has been increased by mean of increasing the number of oscillators. Finally, explanation of the time required to synchronize oscillators in the network at different coupling strengths.
\end{abstract}

Key words

Chaos, Synchronization, Delay, Optoelectronic feedback.

Article info.

Received: Oct. 2015

Accepted: Jan. 2016

Published: Sep. 2016

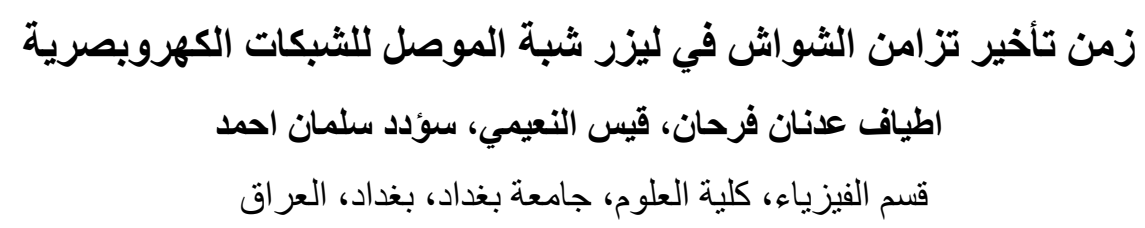

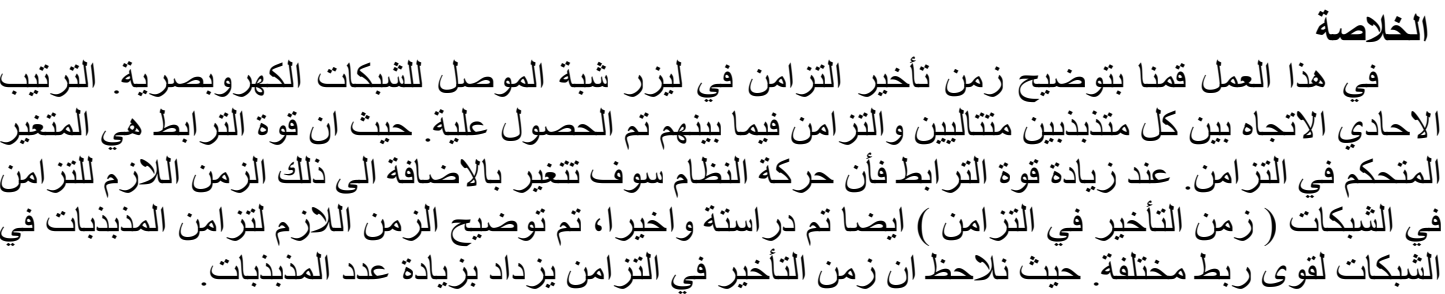

\section{Introduction}

The analysis of synchronization phenomena in the evolution of dynamical systems started in $17^{\text {th }}$ century with the finding of Huygens that weakly coupled pendulum clocks (hanging at the same beam) become synchronized in phase [1]. Synchronization of chaos [2,3] is an adjustment of rhythms of oscillating objects due to their weak interaction. Synchronization of oscillators is a universal and ubiquitous phenomenon in nature [4]. In general, more complicated synchronization may be observed including cluster and group synchronization $[5,6]$. Many aspects of delay dynamics have been observed and studied first in laser systems [7,8]. F. Sorrentino and E. Ott 2007[9] considered two groups of nodes governed by different local dynamics. Jhon F. Martinez Avila and J. R. Rios Leite 2009 [10] explain time delays in the synchronization of chaotic coupled lasers with feedback. Thomas Dahms et al. 2012 study Cluster and group synchronization in delay-coupled networks they derive the master stability function and show the restrictions that arise upon the topology and investigate the 
symmetries that group and cluster synchronization impose on the master stability function. They also demonstrate this symmetry for networks of delay coupled lasers and Multiple coupling matrices are introduced [11].

The unidirectional synchronization, will be investigated in this paper and correlation between successive oscillators was studied. Also we presented here the relation between time required to synchronize and number of oscillators for a single coupling strength and for different coupling strength $\mathrm{k}$.

\section{The dynamical model}

The population inversion for semiconductor lasers (SLs) is replaced by the carrier density $(\mathrm{N})$ produced by electron-hole recombination. The carrier density and the photon number (which is equivalent to the absolute square of the field amplitude) are frequently used as the variables of the rate equations for SLs. However, for the general descriptions of the dynamics in SLs, we must employ the complex amplitude of the field (the amplitude and the phase of the field) instead of the photon number.

The carrier density $\mathrm{N}$ and dynamics of the photon density $\mathrm{S}$ is described by the usual single mode SL rate equations appropriately modified in order to include the ac-coupled optoelectronic feedback [12] [13].

$$
\begin{aligned}
& \dot{S}=\left[g\left(N-N_{t}\right)-\gamma_{0}\right] S \\
& \dot{N}=\frac{I_{0}+f_{F}(I)}{e V}-\gamma_{C} N-g\left(N-N_{t}\right) S \\
& \dot{I}=-\gamma_{f} I+\kappa \dot{S}
\end{aligned}
$$

where $g$ is the differential gain, $\mathrm{N}_{\mathrm{t}}$ is the carrier density at transparency, $\gamma_{o}$ is the photon damping, $I_{o}$ is the bias current, $f_{F}(I) \equiv A I /\left(I+s^{\prime} I\right)$ is the feedback amplifier function, e is the electron charge, $\mathrm{V}$ is the active layer volume, $\gamma_{\mathrm{c}}$ is the population relaxation rate, $\gamma_{\mathrm{f}}$ is the cutoff frequency of the high-pass filter, where $I$ is the highpass-filtered feedback current (before the nonlinear amplifier) and $\kappa$ is a coefficient proportional to the photodetector responsivity.

It is useful to rewrite equations $1,2,3$ in dimensionless form and that for numerical and analytical purposes. So, we introduce the new variables

$$
\begin{aligned}
& x_{i}=\frac{g}{\gamma_{C}} S, \mathcal{Y}_{i}=\frac{g}{\gamma_{0}}\left(N-N_{t}\right), \\
& w_{i}=\frac{g}{\kappa \gamma_{C}} I-x
\end{aligned}
$$

and the time scale $t=\gamma_{0} t$. Then the rate equations can be written as:

$$
\begin{aligned}
& \dot{x}_{i}=x_{i}\left(y_{i}-1\right) \\
& \dot{y}_{i}=\gamma\left(\delta_{0}-y_{i}+f\left(w_{i}+x_{i}\right)-\right. \\
& \left.x_{i} y_{i}\right) \\
& \dot{w}_{i}=-\varepsilon\left(w_{i}+x_{i}\right)
\end{aligned}
$$

where $\quad(i=1,2,3 \ldots 37),\left(w_{i}+x_{i}\right) \equiv$ $\alpha \frac{w_{i}+x_{i}}{1+s\left(w_{i}+x_{i}\right)}, \delta_{0}=\left(I_{0}-I_{t}\right) /$ $\left(I_{t h}-I_{t}\right)$ is the bias current, $\left(I_{t h}=e V \gamma_{C}\left(\frac{\gamma_{0}}{g}+N_{t}\right) \quad\right.$ is the solitary laser threshold current, $\gamma=\frac{\gamma_{C}}{\gamma_{0}}, \varepsilon=$ $\frac{w_{0}}{\gamma_{0}}$ is the bandwidth at resonant frequency $\omega_{0}$, which is the inverse of the familiar quality factor $\mathrm{Q}, \alpha=$ $A \kappa /\left(e V \gamma_{0}\right)$ is the feedback strength, $s=\gamma_{C} s \kappa / g$ is the saturation coefficient. Let $z_{i}=w_{i}+$ $x_{i}$, the previous equations can be written as:

$\dot{x}_{i}=x_{i}\left(y_{i}-1\right)$

$\dot{y}_{i}=\gamma\left(\delta_{0}-y_{i}+\alpha \frac{z_{i}}{1+s z_{i}}-x_{i} y_{i}\right)$

$\dot{z}_{i}=-\varepsilon z_{i}+\dot{x}_{i}$

to be easily handled when processed using Berkeley Madonna (BM) Software. These equations representing the nonlinear dynamical system which produced homoclinic chaos (HC) in SL 
with optoelectronic feedback OEFB. The first equation represents the photon density or the intensity for output laser ray, the second equation represents the dynamics of population inversion, while the third equation represents the feedback which is necessary to produce third degree of freedom then nonlinearity and chaos. Then we modifying equations $(7,8,9)$ to get unidirectional synchronization by adding the term $k *\left(\dot{x}_{1}-\dot{x}_{2}\right)$ to the equation 9 in the second oscillator and added the term $k *\left(\dot{x}_{2}-\dot{x}_{3}\right)$ to the equation 9 in the third oscillator and also added the term $k *\left(\dot{x}_{3}-\dot{x}_{4}\right)$ to the equation 9 in the forth oscillator etc. until we reached to the final oscillator which we want.

\section{Results and discussions}

In this work, we were satisfied the unidirectional synchronization networks, first step between two successive oscillators; noted that at $\mathrm{k}=0$, there is non synchronization was showed in Fig.1a, where coupling strength $\mathrm{k}$ is a control parameter. In addition, when the $\mathrm{k}$ value was increased gradually a partial synchronization at $\mathrm{k}=0.011$, was observed as it shown in Fig. $1 \mathrm{~b}$ and if the gradual increase continued in $\mathrm{k}$ value, full synchronization at $\mathrm{k}=0.019$ was obtained as it shown in Fig.1c.



(a)

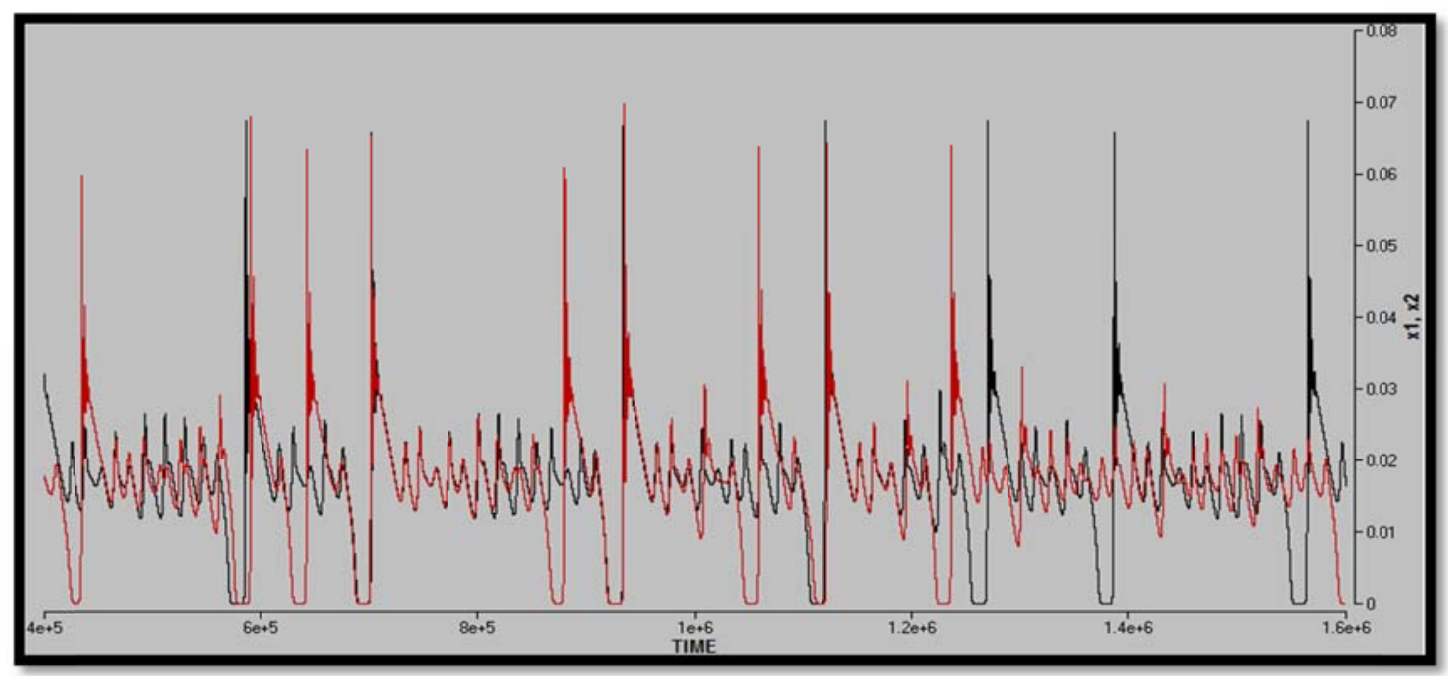

(b)

Fig. 1: (a) Non synchronization between two successive oscillators, at $k=0$. (b) partial synchronization between two successive oscillators, at $k=0.011$. 


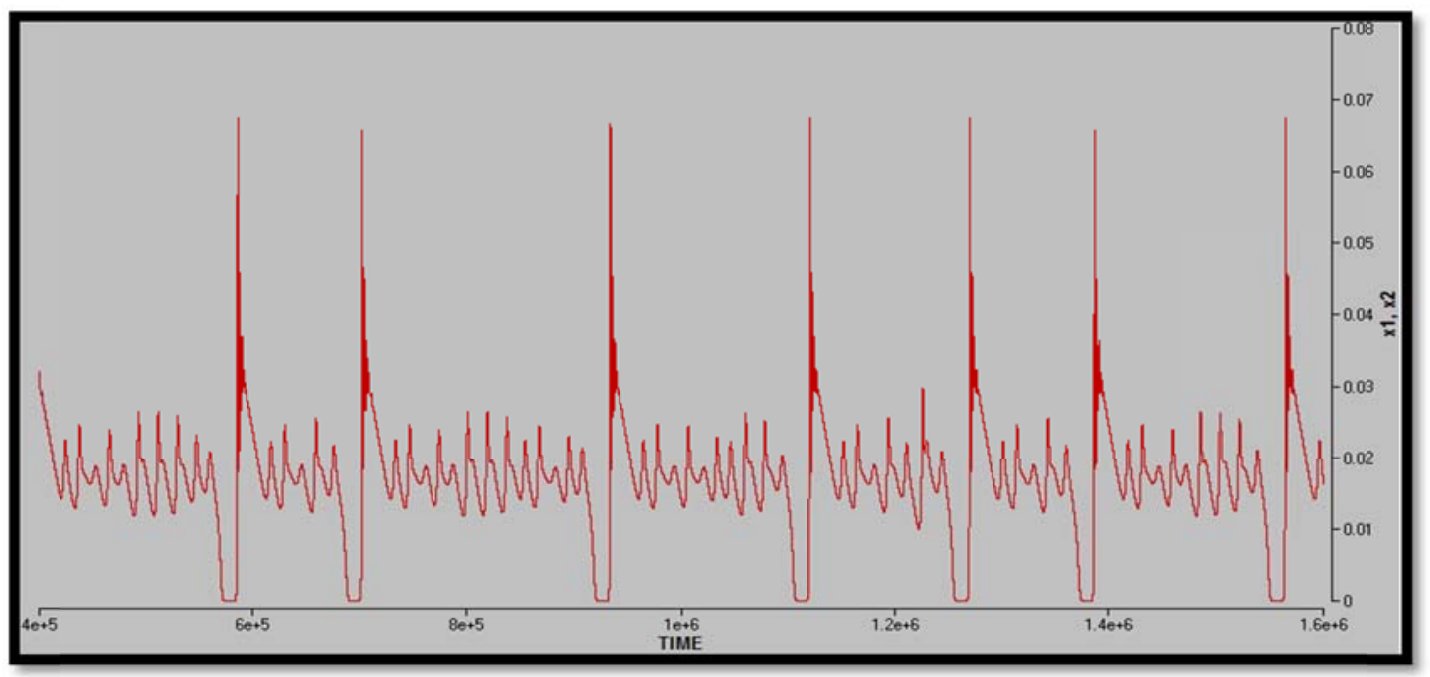

(c)

Fig. 1: (c) full synchronization between two successive oscillators, at $k=0.019$.

In addition, we study the correlation between the two successive oscillators as shown in Fig. 2, and noted from this figure the oscillator one (master) is still constant for all different $\mathrm{k}$ values, but oscillator two (slave) is changed according to the changing in $\mathrm{k}$ values, because $\mathrm{k}$ is the control parameter.

When the $\mathrm{k}$ value is little, there is non synchronization between the two successive oscillators, and that clear because of the large distance between the two successive oscillators as shown in Fig.2.

When we started to gradually increase in $\mathrm{k}$ value, we noted that synchronization also start to increased gradually until it became full synchronization at $\mathrm{k}=0.019$, and one oscillator match with another oscillator as it showed in Fig. 2.

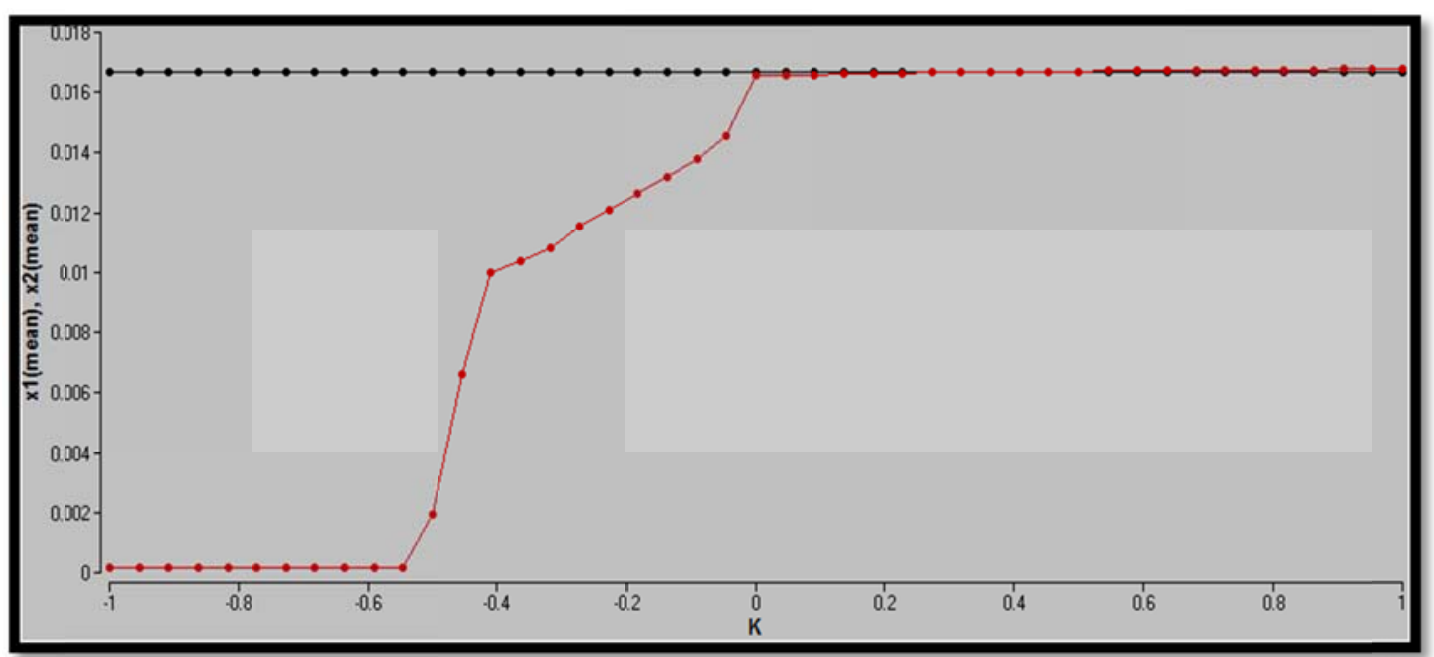

Fig. 2: The relation between the coupling strength $k$ and mean value of the time.

Second step unidirectional oscillators as shown in Fig. 3. synchronization are satisfied for 37 


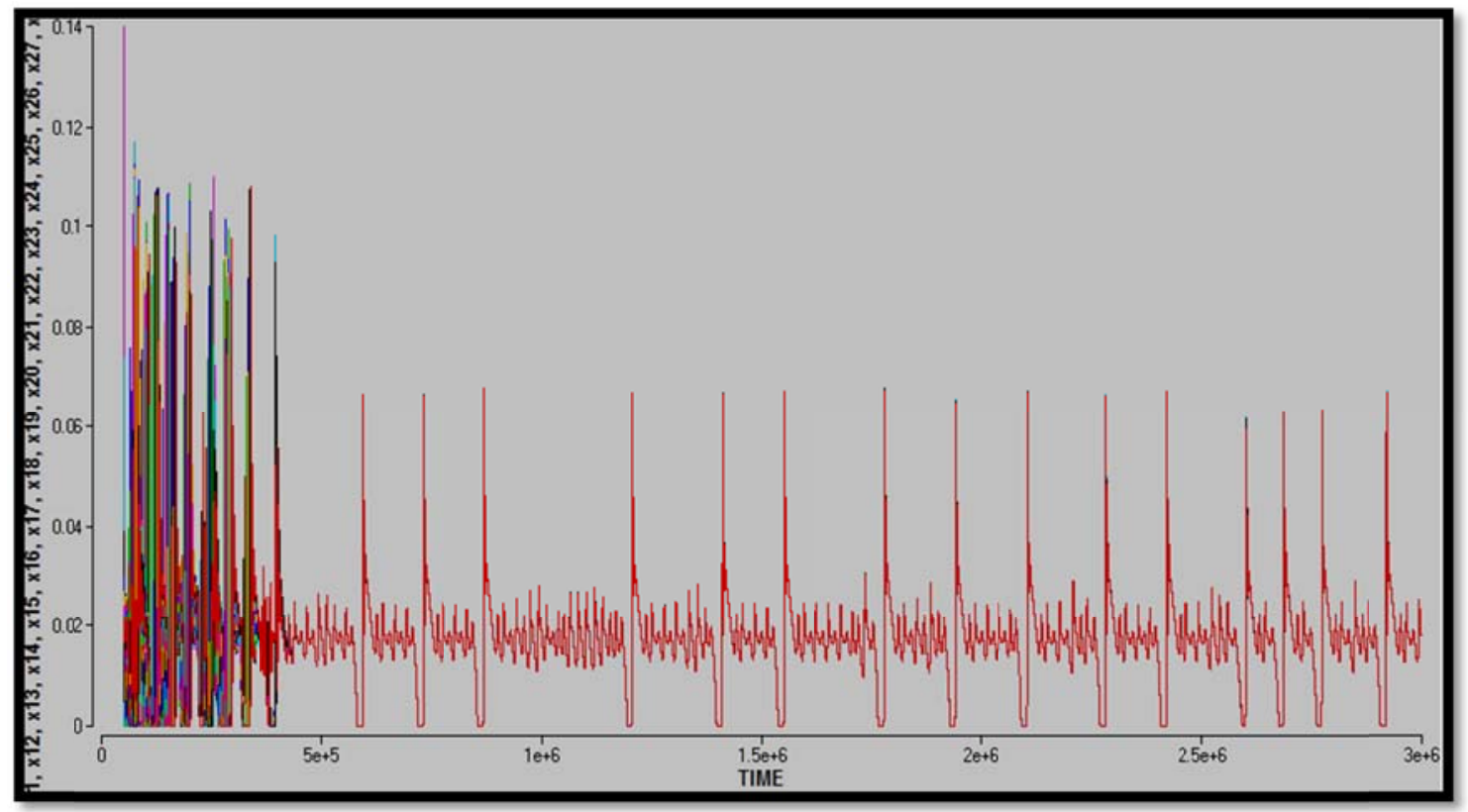

Fig. 3: Open loop unidirectional synchronization for 37 oscillators at $k=0.13$.

From this, we studied the time required to synchronize (delay of synchronization) for the 37 oscillators at $\mathrm{k}=0.12$, as it showed in Fig. 4. It is noted that when we increase the number of oscillators, the time required to synchronize will increase.



Fig. 4: Delay of synchronization for 37 oscillators.

Now, we clarified the time required to synchronize (delay of synchronization) for the 37 oscillators with different $\mathrm{k}$ values $(\mathrm{k}=0.1, \mathrm{k}=0.13$, $\mathrm{k}=0.16, \mathrm{k}=0.19, \mathrm{k}=0.22$ ) as shown in Fig. 5.
If we started to increase the number of oscillators for different five $\mathrm{k}$ values, the time required to synchronize (delay of synchronization) will increase as it showed in Fig. 5. 


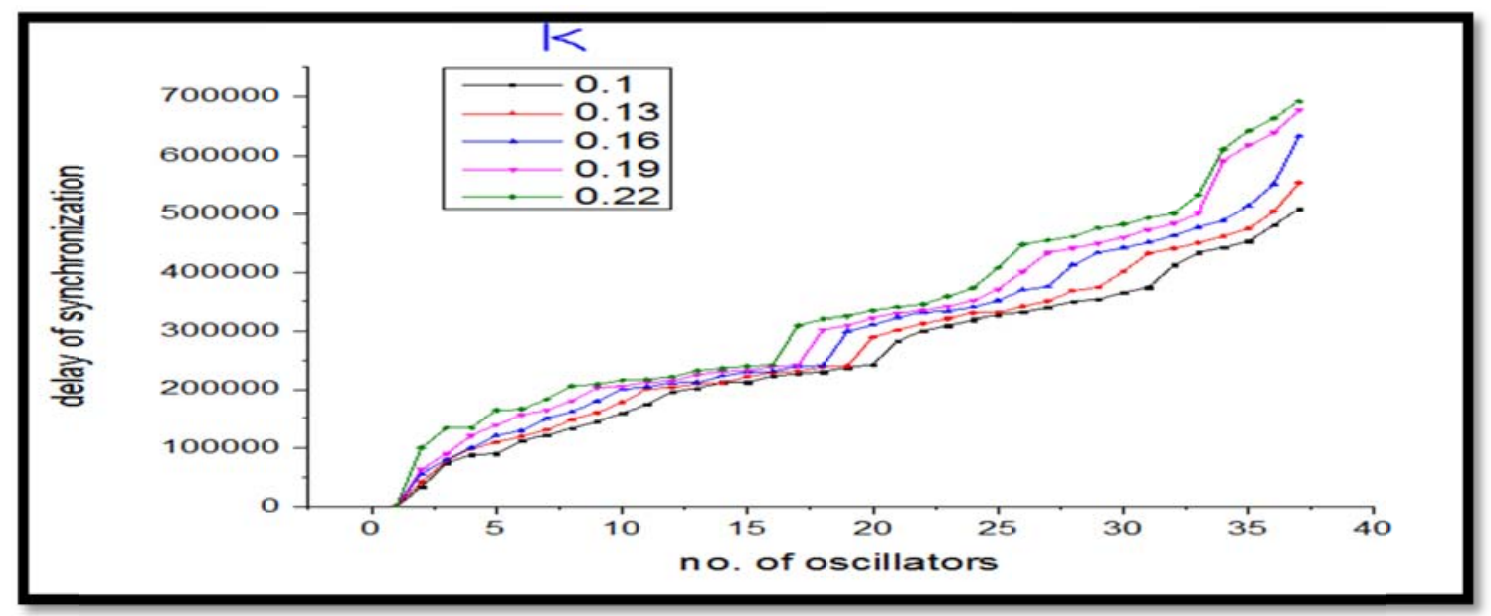

Fig. 5: Delay of synchronization for 37 oscillators with different $k$ values $(k=0.1, k=0.13$, $k=0.16, k=0.19, k=0.22)$.

\section{Conclusions}

To conclude, for unidirectional configuration between successive oscillators if we increase coupling strength, the dynamic of the system has been change, when $\mathrm{k}=0$ we noted there is non synchronization after increase coupling strength $\mathrm{k}$ to 0.011 we get partial synchronization and when we increase coupling strength $\mathrm{k}$ to 0.011 we have full synchronization. We can obtain synchronization between 37 oscillators and study the relation between the number of oscillators and delay of synchronization for a single $\mathrm{k}$ value, different $\mathrm{k}$ value and note that when we increase the number of oscillators delay of synchronization increase too.

\section{References}

[1] C. Hugenii, "Horoloquim Oscilatorium,"Apud F. Muguet (1673).

[2] Tao Deng, Guang-Qiong Xia, Zheng-Mao $\mathrm{Wu}$, Xiao-Dong Lin, JiaGui Wu, Optics Express, 19 (2011) 8762-8773.

[3] Hitoshi Fujino, Junji Ohtsubo, Optical Review, 8 (2001) 351-357.

[4] P. A., Rosemblum, M. Kurths, "Synchronization, A Universal
Concept in Nonlinear Sciences". Cambridge University Press, (2001).

[5] J. Kestler, W. Kinzel, I. Kanter, Phys. Rev. E 76, 3 (2007) 035202.

[6] Y. Aviad, I. Reidler, M. Zigzag, M. Rosenbluh, I. Kanter, Opt. Express, 20 (2012) 4352-4359.

[7] M.C. Soriano, J. Garcia-Ojalvo, C.R. Mirasso, I. Fischer Reviews of Modern Physics, 85 (2013) 421-470.

[8] M. Lakshmanan, D.V. Senthilkumar, Dynamics of Nonlinear Time-Delay Systems, Springer-Verlag: Berlin, Heidelberg, Germany, (2010). [9] F. Sorrentino and E. Ott, Phys. Rev. E 76, 5 (2007) 056114.

[10] Jhon F. Martinez Avila and J. R. Rios Leite, Optics Express, 17, 24 (2009) 21442-21451.

[11] Thomas Dahms, Judith Lehnert, Eckehard Schöll, Physical Review E 86 (2012) 016202 .

[12] K. Al-Naimee, F. Marino, M. Ciszak, R. Meucci, F. T. Arecchi, New Journal of Physics, 122 ( 2009) 11.

[13] K. Al-Naimee, F. Marino, M. Ciszak1, S. F. Abdalahı1, R. Meucci1, F. T. Arecchi, Eur. Phys. J. D, 58 (2010) 187. 